\title{
Right Hemihepatectomy
}

National Cancer Institute

\section{Source}

National Cancer Institute. Right Hemihepatectomy. NCI Thesaurus. Code C51569.

Surgical removal of the right hemisphere of the liver. 